\title{
Estudo radiométrico da Praia da Areia Preta em Anchieta-ES
}

\author{
Braga, H. $\mathrm{M}^{1}$;Ferreira, B.C.S ${ }^{1}$; Passamai, Jr.J.L. ${ }^{2 *}$; \\ 1 Departamento de Química e Física, Universidade Federal do Espírito Santo, Alegre, ES, Brasil. \\ 2 Departamento de Física, Universidade Federal do Espírito Santo, Vitória, ES, Brasil.
}

*e-mail:passamaijr@gmail.com

Resumo

O objetivo do nosso trabalho foi investigar através de uma determinada rota a atividade radioativa na areia Praia da Areia Preta, na cidade de Anchieta-ES utilizando uma metodologia dinâmica (variação com tempo). As medidas foram tomadas em tempos distintos durante o período de (agosto de 2016 a abril de 2017). Para tanto um ciclocomputador Etrex-10 foi usado para determinar uma rota na areia da praia usada por banhistas e também um contador Geiger que mediu e armazenou os dados.

\begin{abstract}
The objective of our work was to investigate, through a determined route, the radioactive activity in the Sand of Areia Preta sand, in the city of Anchieta-ES using a dynamic methodology (variation with time). Measures were taken at different times during the period from (August 2016 to April 2017). An Etrex-10 cyclocomputer was used to determine a route in the beach sand used by bathers and also a Geiger counter that measured and stored the data.
\end{abstract}

\section{Introdução}

Um estudo anterior de Orlando et al. [1] relata a possível relação da atividade radioativa nas praias de Guarapari com efeitos biológicos. Indiferente ao efeito da radiatividade, verificase que em geral os autores, anteriores a este estudo, descrevem as propriedades das areias utilizando amostras retiradas em um único local, de forma singular no tempo [2,3,4]. Esses trabalhos tiram correlações sobre uma amostra singular e com uma composição não reprodutível no tempo.

Neste trabalho foi realizada a medição do nível da atividade radioativa $(\mu \mathrm{Sv} / \mathrm{h})$ presente na praia da Areia Preta em Anchieta variando no tempo, os estudos começaram em agosto de 2016 e foram até abril de 2017. Nesses nove meses de medidas, o intervalo entre as medidas foi de 21 dias.

Para a rota ser seguida várias vezes no mesmo caminho utilizamos as coordenadas de

Tabela 1: Coordenadas da rota utilizada. georreferenciamento de a tabela a seguir.

\begin{tabular}{|c|c|}
\hline $20^{\circ} 50^{\prime} 1.52^{\prime \prime} \mathrm{S}$ & $40^{\circ} 41^{\prime} 44.17^{\prime \prime} \mathrm{W}$ \\
\hline $20^{\circ} 50^{\prime} 0.53^{\prime \prime} \mathrm{S}$ & $40^{\circ} 41^{\prime} 45.56^{\prime \prime} \mathrm{W}$ \\
\hline $20^{\circ} 49^{\prime} 59.98^{\prime \prime} \mathrm{S}$ & $40^{\circ} 41^{\prime} 47.25^{\prime \prime} \mathrm{W}$ \\
\hline $20^{\circ} 49^{\prime} 59.67^{\prime \prime} \mathrm{S}$ & $40^{\circ} 41^{\prime} 48.94^{\prime \prime} \mathrm{W}$ \\
\hline $20^{\circ} 49^{\prime} 59.77^{\prime \prime} \mathrm{S}$ & $40^{\circ} 41^{\prime} 50.75^{\prime \prime} \mathrm{W}$ \\
\hline $20^{\circ} 50^{\prime} 0.05^{\prime \prime} \mathrm{S}$ & $40^{\circ} 41^{\prime} 52.41^{\prime \prime} \mathrm{W}$ \\
\hline $20^{\circ} 50^{\prime} 0.51^{\prime \prime} \mathrm{S}$ & $40^{\circ} 41^{\prime} 54.41^{\prime \prime} \mathrm{W}$ \\
\hline $20^{\circ} 50^{\prime} 1.15^{\prime \prime} \mathrm{S}$ & $40^{\circ} 41^{\prime} 56.15^{\prime \prime} \mathrm{W}$ \\
\hline $20^{\circ} 50^{\prime} 1.77^{\prime \prime} \mathrm{S}$ & $40^{\circ} 41^{\prime} 57.72^{\prime \prime} \mathrm{W}$ \\
\hline $20^{\circ} 50^{\prime} 2.41 " \mathrm{~S}$ & $40^{\circ} 41^{\prime} 59.38^{\prime \prime} \mathrm{W}$ \\
\hline $20^{\circ} 50^{\prime} 3.13^{\prime \prime} \mathrm{S}$ & $40^{\circ} 42^{\prime} 1.03^{\prime \prime} \mathrm{W}$ \\
\hline $20^{\circ} 50^{\prime} 3.91^{\prime \prime} \mathrm{S}$ & $40^{\circ} 42^{\prime} 2.55^{\prime \prime} \mathrm{W}$ \\
\hline $20^{\circ} 50^{\prime} 4.73 " \mathrm{~S}$ & $40^{\circ} 42^{\prime} 4.12^{\prime \prime} \mathrm{W}$ \\
\hline $20^{\circ} 50^{\prime} 5.20^{\prime \prime} \mathrm{S}$ & $40^{\circ} 42^{\prime} 4.90^{\prime \prime} \mathrm{W}$ \\
\hline
\end{tabular}




\section{Resultados e Discussões}

$\mathrm{Na}$ figura 01 , temos as três primeiras medidas realizadas entre o mês de agosto de 2016 até abril de 2017 e obtivemos o seguinte gráfico.

Fig. 01. Gráfico das três primeiras medidas.

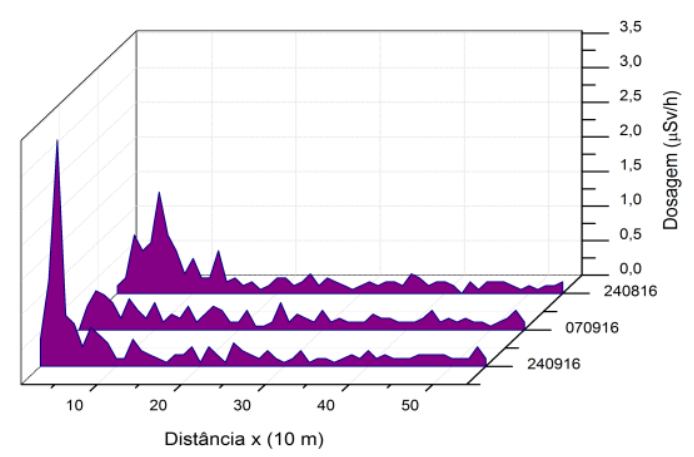

Á direita do gráfico acima temos 3 datas que foram feitas as medidas, a primeira foi feita em 24/08/16 a segunda em 07/09/16 e a terceira em 24/09/16.

Durante a primeira medida verificamos uma dose de aproximadamente $1,46(\mu \mathrm{Sv} / \mathrm{h})$ em $60 \mathrm{~m}$, na segunda medida verificamos uma dose de $0,56(\mu \mathrm{Sv} / \mathrm{h})$ em $30 \mathrm{~m}$, na terceira medida verificamos uma dosagem 3,27 $(\mu \mathrm{Sv} / \mathrm{h})$ em $30 \mathrm{~m}$.

Realizamos mais medidas e obtivemos o gráfico a seguir.

Fig. 02. Gráfico das quatro últimas medidas.

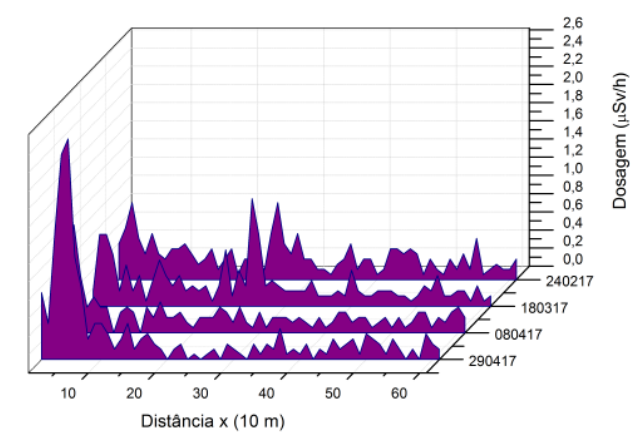

A medida do dia 24/02/17 mostra uma dosagem de $0,84(\mu \mathrm{Sv} / \mathrm{h})$ em $30 \mathrm{~m}$ e $250 \mathrm{~m}$, no dia 18/03/17 mostra a dosagem de 1,18 $(\mu \mathrm{Sv} / \mathrm{h})$ em $250 \mathrm{~m}$, em 08/04/17 fizemos a medida e obtivemos uma dosagem de 1,18 $(\mu \mathrm{Sv} / \mathrm{h})$ em $20 \mathrm{~m}$, em 29/04/17 obtivemos uma dosagem de $2,42(\mu \mathrm{Sv} / \mathrm{h})$ em $50 \mathrm{~m}$.

\section{Conclusões}

Nossas medições neste trabalho mostram que a radiação nesta praia varia de intensidade com o tempo e se desloca no espaço continuamente nesta praia, esta praia apresentou pouca intensidade de radiação.

O ponto a ser ressaltado aqui é de que análises instantâneas de amostras de areia não caracterizam bem a Praia da Areia Preta, uma vez que o sistema é dinâmico.

Somente uma análise temporal com base em 1 ano pode fornecer valores médios da atividade radioativa na Praia da Areia Preta em Anchieta-ES.

\section{Referências}

[1] Orlando, M T D, et al. Correlation between Breast Cancer and Radiation Level of Guarapari City - ES, Blucher Proceedings ISSN: $2358-2359$ V 01 , (2014) n 02 DOI: $10.5151 /$ phypro-ecfa-020

[2] Fujinami, N.; T. Koga, T.; Morishima. H. External Expousure Rates From Terrestrial Radiantion At Guarapari And Meaipe In Brazil. (1999).

[3] Vasconcelos, D.C, Et Al. Modelling Natural Radioactivity In Sand Beaches Of Guarapari, Espírito Santo State, Brazil. (2013).

[4] Calheiro, D.S.; Passamai. Jr.,J.L. Estudo da Radiação na areia da Praia da Areia Preta. (2016). DOI: 10.5151/phypro-vii-efa-035

Agradecimentos.

Comissão Nacional de Energia Nuclear (CNEN), ao CNPQ e a Fundação de Amparo à Pesquisa e Inovação do Espírito Santo (FAPES) (projetos 098/2019 e 270/2019). 
Blucher Proceedings

$1^{\circ}$ Workshop sobre Areias Monazíticas

Meaípe, Guaraparí- ES, Brasil, May 2019 